\title{
Effect of the 2004 Mid Niigata Prefecture Earthquake on Patients with Endocrine Disorders
}

\author{
KYUZI KAMOI, MIDORI TANAKA, TOMOO IKARASHI AND MASASHI MIYAKOSHI
}

The Diabetes and Endocrine \& Metabolism Disease Center, Nagaoka Red Cross Hospital, 297-1 Terajima-machi, Nagaoka, Niigata 940-2085, Japan

\begin{abstract}
A major earthquake (Richter scale magnitude 6.8) struck the Chuetsu district of Niigata Prefecture, Japan, a rural area with mountain villages, on October 23, 2004. Strong aftershocks (Grade 5-6 on the Japanese intensity scale, JIS) continued for 2 months. We analyzed the earthquake's impact on 229 patients with various endocrine disorders [6 central diabetes insipidus (CDI), 16 adrenal insufficiency (AI) including 5 panhypopituitarism, 10 ACTH isolated deficiency and 1 Addison's disease, 145 Graves' disease and 62 Hashimoto's disease]. The status of patients with CDI or AI was not adversely affected by the earthquake. Twenty-eight (19\%) patients with Graves' disease developed more severe hyperthyroidism; the incidence of developing more severe hyperthyroidism increased with greater degrees of hyperthyroidism. Three (5\%) patients with Hashimoto's disease developed increased TSH concentrations. Most patients stayed in their own houses following the first shock. The median PTSD total score for all patients was low. However, the PTSD total score in patients with CDI or Hashimoto's disease was significantly higher than in other patients, while the subscore of mental status in patients with AI was significantly much lower than in other patients. In patients with Hashimoto's disease, patients whose hypothyroidism worsened had higher total and environmental effects score than patients whose hypothyroidism remained stable. Comparing patients whose hyperthyroidism became more severe to those in whom it remained stable, as well as on multiple logistic regression analysis, serum TRAb was found to be a risk factor for developing more severe hyperthyroidism. In conclusion, our findings indicate that Graves' disease patients need to maintain their euthyroid state with a low serum TRAb titer to prevent the development of further thyroid dysfunction after an earthquake, and that all patients should continue to take their medication, since it is likely that their lives will be interrupted by environmental effects owing to earthquake-shock, especially patients with CDI or Hashimoto's disease. Due to the risk of medical facility closure during a disaster, all patients should always have a note or copy of their medical records, including medical history and medications used, to avoid relying on patients remembering their drug names and doses. Furthermore, appropriate information should be provided by all means possible, including the mass media, to affected individuals, particularly those with AI, to decrease the occurrence of adverse consequences.
\end{abstract}

Key words: Earthquake, Hashimoto's disease, Graves' disease, Adrenal insufficiency, Diabetes insipidus, Addison's disease (Endocrine Journal 53: 511-521, 2006)

A sequence of powerful earthquakes struck Mid Niigata Prefecture (Chuetsu area) in central Japan (Mid Niigata Prefecture Earthquake: MNPE) at 5:56 PM on October 23, 2004 [1]. The main shock had a hypocenter located at a depth of $13 \mathrm{~km}$ and had a magnitude of 6.8 on the Richter scale. Using the 7-grade Japanese Intensity

Received: February 1, 2006

Accepted: April 20, 2006

Correspondence to: Dr. Kyuzi KAMOI, the Diabetes and Endocrine \& Metabolism Disease Center, Nagaoka Red Cross Hospital, 297-1 Terajima-machi, Nagaoka, Niigata 940-2085, Japan
Scale (JIS), this earthquake registered $7+$ and the nearest area to the hypocenter recorded a maximum acceleration of 1750 gal. Aftershocks occurred in northeasterly and southwesterly directions for about $30 \mathrm{~km}$.

Although it has been shown that great earthquakes such as Hanshin-Awaji Earthquake (HAE) of 1995 affect many patients with acute and chronic diseases in various ways, as reflected by changes in both morbidity and mortality $[2,3]$, the effects on patients with endocrine disorders other than diabetes mellitus are unclear [2]. It is known that stress has a profound influence on the immune system through many neuroendocrine net- 
works [4], which are essential for survival during periods of stress [5]. One such neuroendocrine network is the hypothalamo-pituitary-adrenal (HPA) axis, in which the corticoid hormone is the main effector at the endpoint of the response [5]. However, it is known that stress and the corticoid hormone have a differential effect on the T-helper/suppressor-cells of the immune system, which are considered to be a cause of Graves' disease [6].

We investigated the impact of the MNPE on patients with various endocrine disorders, including: adrenal insufficiency (AI), as an example of a disturbance of the HPA axis; central diabetes insipidus (CDI), as a comparison with AI; and Graves' and Hashimoto's diseases, as examples of autoimmune thyroid diseases. Furthermore, we also focused our discussion on the factors that contributed to functional deterioration after the MNPE.

\section{Materials and Methods}

\section{Subjects}

This prospective, uncontrolled study lasted for 3 months after the initial shock. We selected 229 patients with endocrine disorders who had attended our clinic every 1 to 3 months before the earthquake.

We enrolled 6 patients with CDI, 16 with AI (5 with panhypopituitarism, 10 with isolated ACTH deficiency, and 1 with Addison's disease), 145 with Graves' disease, and 62 with Hashimoto's disease. AI patients were diagnosed based on the guidelines for hypothalamopituitary disorders [7], and the Graves' disease and Hashimoto's disease patients were diagnosed based on the guidelines of the Japan Thyroid Association [8].

The female to male ratio in the patients with Graves' and Hashimoto's diseases, CDI, and panhypopituitarism was high (Tables 1 and 9), while, in the patients with isolated ACTH deficiency, the female to male ratio was low (Table 9).

The mean age of patients with Graves' disease and CDI was significantly lower than that of patients with Hashimoto's disease and AI (Tables 1 and 9). There was no significant difference in age among the other types of patients.

Of the patients with Graves' disease, $92 \%$ were treated with drugs or ${ }^{131}$ I therapy (Table 1), while $87 \%$ of the patients with Hashimoto's disease were treated with levothyroxine ( $L$-T4) (Table 1). Replacement therapy consisted of: desmopressin acetate (DDAVP) for CDI patients; DDAVP with combination $L$-T4 and hydrocortisone for patients with panhypopituitarism; and hydrocortisone for patients with isolated ACTH deficiency and Addison's disease (Table 9).

\section{Methods}

\section{2-1 Physical and laboratory examination}

Physical and laboratory examinations were done before and within 2 months after the MNPE. In patients with Graves' and Hashimoto's diseases, the concentrations of free triiodothyronine (FT3), free thyroxine (FT4), and TSH, and the titers of thyrotropin receptor antibody (TRAb), thyroid-stimulating antibody (TSAb), thyroglobulin antibody (TgAb), and thyroid peroxidase antibody (TPOAb) in the blood were assessed by commercially available kits. In patients with other disorders, laboratory examinations were done as needed.

Using the American Thyroid Association guidelines for detecting thyroid dysfunction [9], patients with hyperthyroidism were divided into two groups: a mild hyperthyroid/hyperthyroid group and a mild hypothyroid/ hypothyroid group. Mild (subclinical) hyperthyroidism was defined as normal serum FT3 and FT4 levels with a suppressed serum TSH level, and hyperthyroidism was defined as high FT3 and/or FT4 levels with a suppressed serum TSH level. Mild (subclinical) hypothyroidism was defined as normal serum FT3 and FT4 levels with an increased serum TSH level, and hypothyroidism was defined as low serum FT3 and/or FT4 levels with an increased serum TSH level. In patients with Graves' disease, a deterioration of thyroid function after the earthquake was defined as an increase in the severity of hyperthyroidism. In patients with Hashimoto's disease, a deterioration of thyroid function was defined as an increase in the severity of hypothyroidism.

\section{2-2 Assessment by questionnaire}

The JIS intensity grade of the initial earthquake at each patient's home was determined, and a questionnaire was used to assess each patient's lifestyle and medication compliance during the earthquake and the psychological impact when the patient visited our clinic within 2 months after the initial shock. Patients' lifestyles were divided into 2 categories. The first category consisted of 2 items related to housing status after 
the initial shock: whether the patients were living in their own homes or whether they were living in a shelter. The second category assessed the degree of destruction of the patients' houses: non-destroyed, partially destroyed, half destroyed, massively destroyed, or completely destroyed. Relationships between deterioration in the patient's medical status and earthquake intensity, lifestyles, and psychological impact, as determined by the questionnaire, were analyzed.

To investigate the psychological impact of the earthquake, the posttraumatic stress disorder (PTSD) symptom score was employed, using a questionnaire based on the DSM-IV PTSD [10]. The questionnaire consisted of 15 items: 2 items from category A (experienced threat of life, shudder response), 2 items from category B (repetitive images; bad dreams, repetitive thoughts, and upset reminders, and somatic complaints) and 11 items from category $\mathrm{C}$ (emotional avoidance, behavioral avoidance, psychogenic amnesia, diminished interest in activities, emotional numbing, foreshortened future, sleep difficulties, irritability, disability of concentration, hypervigilance and easily startled). Questions answered in the affirmative were given 1 point, and those in the negative, no points. The questionnaire was administered within 2 months of the initial shock. The total score was used as the total PTSD score. Subscoring was used for the combination of categories A and $\mathrm{B}$ to evaluate environmental effects and for category $\mathrm{C}$ to evaluate the patient's mental condition. This same approach has been used to assess the psychological impact of the HAE [11, 12].

\section{2-3 Statistical analysis}

All values are presented as mean $\pm \mathrm{SD}$. Mean values were compared by using paired or unpaired Student's $t$-tests and Welch's correction. Fisher's exact test was used to compare the different levels of earthquake intensity, lifestyle, and psychological impact among the subgroups. The risk factors for thyroid function deterioration after the earthquake were calculated using multiple logistic regression analysis. Values of FT3, FT4, $\mathrm{TSH}$, and TRAb and ${ }^{131} \mathrm{I}$ therapy before the earthquake, and the dose of thionamide, lifestyles, and PTSD score after the shock were used as risk factors. The analysis was carried out using GraphPad Prism (version 4.03, GraphPad Software, San Diego, CA) and the Statistical Package for the Biosciences (SPBS) (version 9.51, Winesteem Institute of Community Medicine, Tokyo, Japan). An alpha value of less than 0.05 was consid- ered to be statistically significant.

\section{Results}

\section{3-1 Evaluation of laboratory data}

Patients continued to come to the clinic up to the $3 \mathrm{rd}$ month after the initial shock. Immediately after the earthquake, we advised AI patients via the press of the need to continue taking hydrocortisone. Two patients who had been receiving $L$-T4 before the MNPE temporarily stopped taking drugs as determined by questionnaire; one was a 37 -year-old female who was on $L$-T4 for hypothyroidism due to ${ }^{131}$ I therapy for Graves' disease and the other was a 38-year-old female who was on $L$-T4 for hypothyroidism due to Hashimoto's disease.

Most medical facilities continued to function and were able to communicate with each other following the MNPE. Thus, some patients who were unable to attend our clinic during the first month after the initial earthquake received their medications from neighboring medical facilities or pharmacies based on faxed prescriptions, and others received prescriptions from our clinic via mail.

There was no change in the clinical status of the CDI or AI patients' status based on the results of physical examination and laboratory testing. None of the patients with $\mathrm{CDI}$ or $\mathrm{AI}$ interrupted their medications after the earthquake. Therefore, the laboratory data for patients with CDI and AI are not presented.

In 145 patients with Graves' disease, the mean FT3, FT4, and TSH concentrations before the earthquake were within normal ranges, but the mean auto-antibody titers, especially the TRAb, were high. None of these mean values were significantly altered after the earthquake (Table 1). There were no cases of thyroid crisis. However, in $28(19 \%)$ patients, thyroid function had deteriorated after the MNPE (Tables 2-5).

In $6(35 \%)$ of the 17 patients who had mild hypothyroidism before the earthquake, thyroid function had deteriorated after the earthquake (Table 2); their mild hypothyroidism changed to mild hyperthyroidism (Table 2). The TRAb titer and the dose of thiamazole given before the earthquake were significantly higher in the group whose thyroid function had deteriorated than in those whose thyroid function remained stable (Table 2). There were no significant differences in age, FT3, FT4, TSH, TSAb, TgAb, and TPOAb levels, and treatment between the two groups. 
Table 1. Characteristics of patients with Graves' disease and with Hashimoto's disease before and within 2 months after the earthquake

\begin{tabular}{|c|c|c|c|c|c|}
\hline & \multicolumn{2}{|c|}{ Graves' disease } & \multicolumn{2}{|c|}{ Hashimoto's disease } & \multirow{2}{*}{ Normal range } \\
\hline & Before & After & Before & After & \\
\hline Number & 145 & 145 & 62 & 62 & \\
\hline Age (year) & $48 \pm 15$ & & $54 \pm 14^{\#}$ & & \\
\hline $\operatorname{Sex}(F / M)$ & $120 / 25$ & & $57 / 5$ & & \\
\hline Free T3 $(\mathrm{pg} / \mathrm{ml})$ & $3.75 \pm 4.19$ & $3.56 \pm 3.03$ & $2.59 \pm 0.76$ & $2.88 \pm 1.12 *$ & $2.00-4.90$ \\
\hline Free T4 (ng/dl) & $1.65 \pm 1.40$ & $1.52 \pm 0.94$ & $1.32 \pm 0.39$ & $1.37 \pm 0.46$ & $0.70-1.80$ \\
\hline $\mathrm{TSH}(\mu \mathrm{IU} / \mathrm{ml})$ & $3.25 \pm 9.76$ & $3.55 \pm 9.54$ & $4.20 \pm 11.6$ & $2.23 \pm 3.70$ & $0.30-4.30$ \\
\hline TRAb (IU/l) & $28.1 \pm 98.5$ & $30.3 \pm 110$ & $1.0 \pm 0.1$ & $1.0 \pm 0.1$ & $\leq 1.0$ \\
\hline TSAb (\%) & $429 \pm 516$ & $376 \pm 454$ & $140 \pm 134$ & $17 \pm 59$ & $\leq 180$ \\
\hline $\operatorname{TgAb}(\mathrm{U} / \mathrm{ml})$ & $30.1 \pm 99.6$ & $30.4 \pm 113$ & $234 \pm 704$ & $198 \pm 614$ & $\leq 0.3$ \\
\hline TPOAb (U/ml) & $452 \pm 1000$ & $299 \pm 686$ & $741 \pm 1498$ & $628 \pm 1402$ & $\leq 0.3$ \\
\hline Therapy & $134(92)$ & $136(94)$ & $54(87)$ & $54(87)$ & \\
\hline Thionamide & $67(46)$ & $69(48)$ & & & \\
\hline Thionamide and levothyroxine & $9(6)$ & $10(7)$ & & & \\
\hline${ }^{131}$ I therapy & $44(37)$ & $55(38)$ & & & \\
\hline${ }^{131} \mathrm{I}$ and thionamide & $4(3)$ & $4(3)$ & & & \\
\hline${ }^{131} \mathrm{I}$ and levothyroxine & $31(21)$ & $32(22)$ & & & \\
\hline Levothyroxine & $13(9)$ & $12(8)$ & $54(87)$ & $54(87)$ & \\
\hline
\end{tabular}

* $\mathrm{P}<0.01$ vs. before. ${ }^{\#} \mathrm{P}<0.01$ vs. Graves' disease. Parenthetical number represents the percentage of each item in each group.

Table 2. Characteristics in 17 mild hypothyroid patients with Graves' disease worsened thyroid function occurred within 2 months after the earthquake compared with values before it

\begin{tabular}{|c|c|c|c|c|c|}
\hline & \multicolumn{2}{|c|}{ Non-worsened group } & \multicolumn{2}{|c|}{ Worsened group } & \multirow{2}{*}{ Normal range } \\
\hline & Before & After & Before & After & \\
\hline Number & $11(65)$ & $11(65)$ & $6(35)$ & $6(35)$ & \\
\hline Age (year) & $47 \pm 17$ & & $46 \pm 15$ & & \\
\hline $\operatorname{Sex}(F / M)$ & $6 / 5$ & & $4 / 2$ & & \\
\hline Free T3 $(\mathrm{pg} / \mathrm{ml})$ & $2.35 \pm 0.47$ & $2.63 \pm 0.32$ & $2.25 \pm 0.80$ & $4.68 \pm 3.60$ & $2.00-4.90$ \\
\hline Free T4 (ng/dl) & $0.85 \pm 0.36$ & $1.13 \pm 0.26^{*}$ & $0.80 \pm 0.54$ & $1.74 \pm 0.56^{*, \$}$ & $0.70-1.80$ \\
\hline $\mathrm{TSH}(\mu \mathrm{IU} / \mathrm{ml})$ & $17.4 \pm 23.5$ & $4.82 \pm 3.79 *$ & $24.5 \pm 21.9$ & $0.08 \pm 0.07 * \$$ & $0.30-4.30$ \\
\hline TRAb (IU/l) & $4.5 \pm 3.0$ & $3.6 \pm 2.7$ & $57 \pm 63^{\$}$ & $59 \pm 92^{\$}$ & $\leq 1.0$ \\
\hline TSAb (\%) & $361 \pm 141$ & $283 \pm 1108$ & $526 \pm 614$ & $495 \pm 481$ & $\leq 180$ \\
\hline $\operatorname{TgAb}(\mathrm{U} / \mathrm{ml})$ & $25 \pm 22$ & & $197 \pm 315$ & & $\leq 0.3$ \\
\hline TPOAb (U/ml) & $796 \pm 210$ & & $1803 \pm 2077$ & & $\leq 0.3$ \\
\hline Therapy & $10(91)$ & $10(91)$ & $6(100)$ & $6(100)$ & \\
\hline Thiamazole & $6(55)$ & $6(55)$ & $4(57)$ & $4(57)$ & \\
\hline Dose (mg/day) & $9 \pm 6$ & $10 \pm 5$ & $18 \pm 3^{\$}$ & $11 \pm 6$ & \\
\hline Levothyroxine & $3(27)$ & $4(36)$ & $3(50)$ & $4(67)$ & \\
\hline Dose ( $\mu \mathrm{g} /$ day $)$ & $67 \pm 29$ & $63 \pm 25$ & $94 \pm 43$ & $105 \pm 45$ & \\
\hline${ }^{131}$ I therapy & $4(36)$ & $4(36)$ & $3(50)$ & $3(50)$ & \\
\hline
\end{tabular}

$* \mathrm{P}<0.001$ vs. before. ${ }^{\$} \mathrm{P}<0.03$ vs. Non-worsened group. Numerals in parenthese of number and therapy items represent the percentage of 18 mild hypothyroid patients with Graves' disease and each group, respectively.

Seven $(9 \%)$ of 76 patients who were euthyroid before the earthquake developed hyperthyroidism, while the others remained euthyroid (Table 3). The TRAb titer and the dose of thiamazole given before the shock were significantly higher in the group who became hyperthyroid than in those who remained euthyroid (Table 3). There were no significant differences in age, FT3, FT4, TSH, TSAb, TgAb, and TPOAb levels, and treatment between the two groups.

Six $(22 \%)$ of 27 patients who had mild hyperthyroid- 
Table 3. Characteristics in 76 euthyroid patients with Graves' disease worsened thyroid function occurred within 2 months after the earthquake compared with values before it

\begin{tabular}{|c|c|c|c|c|c|}
\hline & \multicolumn{2}{|c|}{ Non-worsened group } & \multicolumn{2}{|c|}{ Worsened group } & \multirow{2}{*}{ Normal range } \\
\hline & Before & After & Before & After & \\
\hline Number & $69(91)$ & $69(91)$ & $7(9)$ & $7(9)$ & \\
\hline Age (year) & $49 \pm 14$ & & $42 \pm 17$ & & \\
\hline $\operatorname{Sex}(F / M)$ & $64 / 5$ & & $7 / 0$ & & \\
\hline Free T3 $(\mathrm{pg} / \mathrm{ml})$ & $2.45 \pm 0.33$ & $2.50 \pm 0.40$ & $2.84 \pm 0.35$ & $4.71 \pm 2.27$ & $2.00-4.90$ \\
\hline Free T4 (ng/dl) & $1.27 \pm 0.32$ & $1.24 \pm 0.33$ & $1.19 \pm 0.33$ & $2.02 \pm 0.50 *, \$$ & $0.70-1.80$ \\
\hline $\mathrm{TSH}(\mu \mathrm{IU} / \mathrm{ml})$ & $1.66 \pm 1.04$ & $3.67 \pm 6.88^{*}$ & $1.69 \pm 1.61$ & $0.03 \pm 0.04 *, \$$ & $0.30-4.30$ \\
\hline TRAb (IU/l) & $10 \pm 27$ & $10 \pm 29$ & $145 \pm 361^{\$}$ & $61 \pm 121^{\$}$ & $\leq 1.0$ \\
\hline TSAb (\%) & $140 \pm 135$ & $140 \pm 135$ & $332 \pm 275$ & $318 \pm 280$ & $\leq 180$ \\
\hline $\operatorname{TgAb}(\mathrm{U} / \mathrm{ml})$ & $17 \pm 61$ & $27 \pm 118$ & $44 \pm 71$ & & $\leq 0.3$ \\
\hline TPOAb (U/ml) & $272 \pm 624$ & $309 \pm 672$ & $2224 \pm 175$ & $\leq 0.3$ & \\
\hline Therapy & $60(87)$ & $60(87)$ & $7(100)$ & $7(100)$ & \\
\hline Thionamide & $27(39)$ & $27(39)$ & $5(71)$ & $5(71)$ & \\
\hline Thiamazole (mg/day) & $6 \pm 3$ & $6 \pm 3$ & $15 \pm 11^{\$}$ & $9.5 \pm 8.4$ & \\
\hline Propylthiouracil (mg/day) & $81 \pm 38$ & $81 \pm 38$ & & & \\
\hline Levothyroxine & $36(52)$ & $43(62)$ & $3(43)$ & $3(43)$ & \\
\hline Dose ( $\mu \mathrm{g} /$ day $)$ & $75 \pm 29$ & $73 \pm 35$ & $83 \pm 29$ & $83 \pm 29$ & \\
\hline${ }^{131}$ I therapy & $22(32)$ & $22(32)$ & $2(29)$ & $2(29)$ & \\
\hline
\end{tabular}

$* \mathrm{P}<0.001$ vs. before. ${ }^{\$} \mathrm{P}<0.02$ vs. Non-worsened group. Numerals in parenthese of number and therapy items represent the percentage of 76 euthyroid patients with Graves' disease and each group, respectively.

Table 4. Characteristics in 27 mild hyperthyroid patients with Graves' disease worsened thyroid function occurred within 2 months after the earthquake compared with values before it

\begin{tabular}{|c|c|c|c|c|c|}
\hline & \multicolumn{2}{|c|}{ Non-worsened group } & \multicolumn{2}{|c|}{ Worsened group } & \multirow{2}{*}{ Normal range } \\
\hline & Before & After & Before & After & \\
\hline Number & $21(78)$ & $21(78)$ & $6(22)$ & $6(22)$ & \\
\hline Age (year) & $51 \pm 14^{\#}$ & & $48 \pm 15$ & & \\
\hline $\operatorname{Sex}(F / M)$ & $12 / 9$ & & $4 / 2$ & & \\
\hline Free T3 (pg/ml) & $2.91 \pm 0.56$ & $2.58 \pm 0.85$ & $3.41 \pm 0.77$ & $6.14 \pm 2.30 *, \$$ & $2.00-4.90$ \\
\hline Free T4 (ng/dl) & $1.25 \pm 0.29$ & $1.02 \pm 0.37$ & $1.37 \pm 0.36$ & $2.33 \pm 0.45^{*, \$}$ & $0.70-1.80$ \\
\hline $\mathrm{TSH}(\mu \mathrm{IU} / \mathrm{ml})$ & $0.11 \pm 0.37$ & $7.93 \pm 21.0$ & $0.07 \pm 0.09$ & $0.01 \pm 0.00^{\$}$ & $0.30-4.30$ \\
\hline TRAb (IU/l) & $11 \pm 12$ & $14 \pm 22$ & $38 \pm 48^{\$}$ & $34 \pm 39^{\$}$ & $\leq 1.0$ \\
\hline TSAb (\%) & $576 \pm 525$ & $435 \pm 439$ & $377 \pm 300$ & $409 \pm 410$ & $\leq 180$ \\
\hline $\operatorname{TgAb}(\mathrm{U} / \mathrm{ml})$ & $5.6 \pm 7.2$ & & $3.2 \pm 2.0$ & & $\leq 0.3$ \\
\hline TPOAb (U/ml) & $409 \pm 874$ & & $62 \pm 89$ & & $\leq 0.3$ \\
\hline Therapy & $20(95)$ & $20(95)$ & $4(67)$ & $5(83)$ & \\
\hline Thionamide & $13(62)$ & $13(62)$ & $4(67)$ & $5(83)$ & \\
\hline Thiamazole (mg/day) & $17 \pm 10$ & $11 \pm 7$ & $6.3 \pm 2.3$ & $8.0 \pm 4.5$ & \\
\hline Propylthiouracil (mg/day) & $200 \pm 0$ & $200 \pm 0$ & & & \\
\hline Levothyroxine & $4(19)$ & $4(19)$ & $1(10)$ & $1(10)$ & \\
\hline Dose $(\mu \mathrm{g} /$ day $)$ & $100 \pm 35$ & $94 \pm 43$ & 50 & 50 & \\
\hline${ }^{131}$ I therapy & $9(43)$ & $9(43)$ & $0(0)$ & $0(0)$ & \\
\hline
\end{tabular}

* $\mathrm{P}<0.001$ vs. before. ${ }^{\$} \mathrm{P}<0.02$ vs. Non-worsened group. Numerals in parenthese of number and therapy items represent the percentage of 27 mild hyperthyroid patients with Graves' disease and each group, respectively.

ism before the earthquake became overt hyperthyroid after the earthquake, while the others remained mildly hyperthyroid (Table 4). The TRAb titer was signifi- cantly higher in the group whose thyroid function had deteriorated, as compared to those who remained mildly hyperthyroid (Table 4). There were no significant 
Table 5. Characteristics in 25 hyperthyroid patients with Graves' disease worsened thyroid function occurred within 2 months after the earthquake compared with values before it

\begin{tabular}{|c|c|c|c|c|c|}
\hline & \multicolumn{2}{|c|}{ Non-worsened group } & \multicolumn{2}{|c|}{ Worsened group } & \multirow{2}{*}{ Normal range } \\
\hline & Before & After & Before & After & \\
\hline Number & $16(64)$ & $16(64)$ & $9(36)$ & $9(36)$ & \\
\hline Age (year) & $40 \pm 13$ & & $44 \pm 18$ & & \\
\hline $\operatorname{Sex}(F / M)$ & $15 / 1$ & & $9 / 0$ & & \\
\hline Free T3 $(\mathrm{pg} / \mathrm{ml})$ & $10.8 \pm 8.26$ & $5.64 \pm 4.80$ & $6.70 \pm 7.35$ & $8.02 \pm 7.22 *$ & $2.00-4.90$ \\
\hline Free T4 (ng/dl) & $4.13 \pm 2.53$ & $2.16 \pm 1.46^{*}$ & $3.14 \pm 1.91$ & $3.12 \pm 1.96^{*}$ & $0.70-1.80$ \\
\hline $\mathrm{TSH}(\mu \mathrm{IU} / \mathrm{ml})$ & $0.01 \pm 0.01$ & $2.19 \pm 5.02$ & $0.01 \pm 0.01$ & $0.01 \pm 0.01^{\$}$ & $0.30-4.30$ \\
\hline TRAb (IU/l) & $28 \pm 33$ & $46 \pm 75$ & $43 \pm 44$ & $47 \pm 42$ & $\leq 1.0$ \\
\hline TSAb (\%) & $709 \pm 513$ & $870 \pm 490$ & $638 \pm 574$ & $859 \pm 720$ & $\leq 180$ \\
\hline $\operatorname{TgAb}(\mathrm{U} / \mathrm{ml})$ & $8.8 \pm 9.6$ & & $97 \pm 168$ & & $\leq 0.3$ \\
\hline TPOAb (U/ml) & $487 \pm 108$ & & $674 \pm 1347$ & & $\leq 0.3$ \\
\hline Therapy & $16(100)$ & $16(100)$ & $9(100)$ & $9(100)$ & \\
\hline Thionamide & $9(38)$ & $9(38)$ & $2(22)$ & $2(22)$ & \\
\hline Thiamazole (mg/day) & $12 \pm 8$ & $12 \pm 8$ & $7.5 \pm 3.5$ & $10 \pm 0$ & \\
\hline Propylthiouracil (mg/day) & 300 & 200 & 0 & 0 & \\
\hline Levothyroxine & 1 & 1 & 2 & 2 & \\
\hline Dose ( $\mu \mathrm{g} /$ day $)$ & 100 & 100 & $113 \pm 18$ & $113 \pm 18$ & \\
\hline${ }^{131}$ I therapy & 7 & 7 & 7 & 7 & \\
\hline
\end{tabular}

$* \mathrm{P}<0.001$ vs. before. ${ }^{\$} \mathrm{P}<0.02$ vs. Non-worsened group. Numerals in parenthese of number and therapy items represent the percentage of 25 hyperthyroid patients with Graves' disease and each group, respectively.

differences in age, FT3, FT4, TSH, TSAb, TgAb, and TPOAb levels, and treatment between the two groups.

In $9(36 \%)$ of 25 patients who were hyperthyroid before the earthquake, thyroid function had deteriorated after the earthquake (Table 5); serum FT3 levels increased after the earthquake in these patients, while in the remainder, serum FT3 levels decreased after the earthquake (Table 5). The TRAb titer was higher in the group whose thyroid function had deteriorated (Table 5). There were no significant differences in age, FT3, FT4, TSH, TSAb, TgAb, and TPOAb levels, and treatment between the two groups.

In the patients with Hashimoto's disease, the mean FT3, FT4, and TSH concentrations were within the normal ranges. The TRAb titer in all patients was negative, while the mean $\mathrm{TgAb}$ and TPOAb titers were high (Table 1). All of the values, except for the FT3 concentration, were not significantly changed after the earthquake; the increased FT3 concentration was still within the normal range (Table 1). However, $3(5 \%)$ of 62 patients with Hashimoto's disease who had been euthyroid became mildly hypothyroid after the earthquake (Table 6); the other patients continued to be euthyroid after the earthquake, although serum FT3 levels increased significantly after the earthquake. There was no significant difference in the $L$-T4 dose before and after the earthquake in the group whose thyroid function had deteriorated, and there was no significant difference in the $L$-T4 dose between the two groups after the earthquake.

\section{3-2 Evaluation of complications}

No cases of medical crisis were observed. In the patients with CDI or AI, there was no change in the clinical status of the patients' disorders based on physical examination and laboratory testing. CDI patients had no complaints of polyuria or polydipsia after the earthquake.

\section{3-3 Evaluation of earthquake intensity, lifestyle, and psychological impact}

The median JIS showed that all patients had lived in areas with very strong aftershocks (Tables 7-9).

There was no significant difference in the median JIS between patients with Graves' disease and Hashimoto's disease (Tables 7 and 8). Also, there was no significant difference in the median JIS among the subgroups in whom thyroid function had deteriorated. In all groups, most patients stayed in their own houses after the initial shock (Tables 7-9), while about $25 \%$ of patients with thyroid diseases transiently sought refuge in other houses. The ratio of patients remaining in their own 
Table 6. Characteristics in patients worsened thyroid function with 62 Hashimoto's disease occurred within 2 months after the earthquake compared with values before it

\begin{tabular}{|c|c|c|c|c|c|}
\hline & \multicolumn{2}{|c|}{ Non-worsened group } & \multicolumn{2}{|c|}{ Worsened group } & \multirow{2}{*}{ Normal range } \\
\hline & Before & After & Before & After & \\
\hline Number & $59(95)$ & $58(95)$ & $3(5)$ & $3(5)$ & \\
\hline Age (year) & $54 \pm 15$ & & $58 \pm 6$ & & \\
\hline $\operatorname{Sex}(F / M)$ & $54 / 5$ & & $3 / 0$ & & \\
\hline Free T3 (pg/ml) & $2.60 \pm 0.78$ & $2.89 \pm 1.15^{*}$ & $2.48 \pm 0.31$ & $2.44 \pm 0.22$ & $2.00-4.90$ \\
\hline Free T4 (ng/dl) & $1.33 \pm 0.40$ & $1.37 \pm 0.47$ & $1.26 \pm 0.24$ & $1.22 \pm 0.14$ & $0.70-1.80$ \\
\hline $\mathrm{TSH}(\mu \mathrm{IU} / \mathrm{ml})$ & $4.25 \pm 11.9$ & $1.86 \pm 3.37^{*}$ & $3.19 \pm 1.23$ & $9.42 \pm 2.37 *, \$$ & $0.30-4.30$ \\
\hline TRAb (IU/l) & $1.0 \pm 0.1$ & $1.0 \pm 0.0$ & $1.0 \pm 0.0$ & $1.0 \pm 0.0$ & $\leq 1.0$ \\
\hline TSAb (\%) & $141 \pm 136$ & $117 \pm 60$ & $104 \pm 16$ & $99 \pm 23$ & $\leq 180$ \\
\hline $\operatorname{TgAb}(\mathrm{U} / \mathrm{ml})$ & $240 \pm 720$ & $201 \pm 634$ & $128 \pm 183$ & $165 \pm 281$ & $\leq 0.3$ \\
\hline TPOAb (U/ml) & $740 \pm 1526$ & $600 \pm 1412$ & $748 \pm 923$ & $1010 \pm 1457$ & $\leq 0.3$ \\
\hline Therapy & $52(88)$ & $55(92)$ & $2(67)$ & $2(67)$ & \\
\hline Levothyroxine ( $\mu \mathrm{g} /$ day) & $95 \pm 38$ & $93 \pm 38$ & $63 \pm 18$ & $63 \pm 18$ & \\
\hline
\end{tabular}

$* \mathrm{P}<0.02$ vs. before. ${ }^{\$} \mathrm{P}<0.02$ vs. Non-worsened group. Numerals in parenthesis of number and therapy items represent the percentage of 62 patients with Hashimoto's disease and each group, respectively.

Table 7. Intensity grade of earthquake, lifestyles and psychological aspects in patients associated worsened thyroid function with Graves' disease occurred within 2 months after the earthquake

\begin{tabular}{|c|c|c|c|c|c|c|c|c|c|}
\hline & \multirow[b]{2}{*}{ All } & \multicolumn{2}{|c|}{ Mild hypothyroid } & \multicolumn{2}{|c|}{ Euthyroid } & \multicolumn{2}{|c|}{ Mild hyperthyroid } & \multicolumn{2}{|c|}{ Hyperthyroid } \\
\hline & & $\begin{array}{c}\text { Non- } \\
\text { worsened }\end{array}$ & Worsened & $\begin{array}{c}\text { Non- } \\
\text { worsened }\end{array}$ & Worsened & $\begin{array}{c}\text { Non- } \\
\text { worsened }\end{array}$ & Worsened & $\begin{array}{c}\text { Non- } \\
\text { worsened }\end{array}$ & Worsenec \\
\hline Number & 145 & 11 & 6 & 69 & 7 & 21 & 6 & 16 & 9 \\
\hline Intensity of earthquake (JIS) & $5.5 \pm 0.8$ & $5.7 \pm 0.6$ & $5.5 \pm 0.5$ & $5.6 \pm 0.7$ & $4.9 \pm 2.2$ & $5.5 \pm 0.5$ & $5.5 \pm 0.8$ & $5.1 \pm 0.7$ & $5.6 \pm 0.7$ \\
\hline \multicolumn{10}{|l|}{ Lifestyle Category 1} \\
\hline Own home & $110(75)$ & $9(71)$ & $4(66)$ & $55(80)$ & $5(71)$ & $13(62)$ & $4(67)$ & $14(88)$ & $6(67)$ \\
\hline Refuge house & $35(25)$ & $2(33)$ & $2(34)$ & $14(20)$ & $2(29)$ & $8(38)$ & $2(33)$ & $2(12)$ & $3(33)$ \\
\hline \multicolumn{10}{|l|}{ Lifestyle Category 2} \\
\hline Non-destroyed own house & $37(26)$ & $3(27)$ & $2(34)$ & $14(20)$ & 0 & $6(29)$ & $1(17)$ & $7(44)$ & $3(33)$ \\
\hline Partially destroyed own house & $95(65)$ & $7(72)$ & $3(50)$ & $49(72)$ & $5(71)$ & $14(67)$ & $4(67)$ & $9(56)$ & $5(56)$ \\
\hline Half destroyed own house & $7(5)$ & $1(1)$ & 0 & $2(6)$ & $2(29)$ & $1(4)$ & $1(17)$ & 0 & 0 \\
\hline Massive destroyed own house & $2(1)$ & 0 & 0 & $2(6)$ & 0 & 0 & 0 & 0 & 0 \\
\hline Completely destroyed own house & $4(3)$ & 0 & $1(26)$ & $2(6)$ & 0 & 0 & 0 & 0 & $1(11)$ \\
\hline PTSD total score & $1.5 \pm 1.7$ & $2.1 \pm 1.5$ & $2.0 \pm 2.0$ & $1.3 \pm 1.5$ & $0.9 \pm 1.1$ & $2.0 \pm 2.4$ & $1.3 \pm 2.8$ & $1.6 \pm 1.8$ & $0.9 \pm 1.1$ \\
\hline Category $\mathrm{A}+\mathrm{B}$ & $0.9 \pm 0.8$ & $1.3 \pm 0.9$ & $1.3 \pm 1.2$ & $0.7 \pm 0.8$ & $0.7 \pm 0.8$ & $1.0 \pm 0.8$ & $0.5 \pm 0.8$ & $1.0 \pm 0.8$ & $0.6 \pm 0.5$ \\
\hline Category C & $0.6 \pm 1.2$ & $0.8 \pm 0.9$ & $0.7 \pm 0.8$ & $0.6 \pm 1.6$ & $0.2 \pm 0.4$ & $1.0 \pm 1.8$ & $0.8 \pm 2.8$ & $0.6 \pm 1.3$ & $0.3 \pm 0.7$ \\
\hline
\end{tabular}

The number in parenthese represents the percentage of each item in each group.

houses was not statistically different between the patients with Graves' and those with Hashimoto's diseases (Tables 7 and 8). Most houses did not suffer much damage. There was no difference in the damage sustained between the group whose thyroid function had deteriorated and the group whose thyroid function remained stable.

The mean PTSD total score was low in all patients, except in Hashimoto's disease patients, in whom the mean PTSD total score was significantly higher, especially in patients whose thyroid function had deterio- rated, and in CDI patients (Tables 7-9). In patients with Hashimoto's disease, the mean PTSD total score was significantly higher in patients whose thyroid function had deteriorated than in those in whom thyroid function remained stable (Table 8). There was no significant difference in the median PTSD total score between patients with CDI and patients with Hashimoto's disease. Also, there was no significant difference in the median PTSD total score among the other groups.

The mean score of the combined categories A and B in Hashimoto's disease and AI patients was significant- 
Table 8. Intensity grade of earthquake, lifestyles and psychological aspects in patients worsened thyroid function with Hashimoto's diseases occurred within 2 months after the earthquake

\begin{tabular}{lccc}
\hline & All & Non-worsened & Worsened \\
\hline Number & 62 & 59 & 3 \\
Intensity of earthquake (JIS) & $5.5 \pm 0.7$ & $5.5 \pm 0.6$ & $5.3 \pm 2.0$ \\
Lifestyle Category 1 & $47(76)$ & $45(76)$ & $2(66)$ \\
$\quad$ Own home & $15(24)$ & $14(24)$ & $1(34)$ \\
$\quad$ Refuge house & $12(19)$ & $12(20)$ & 0 \\
Lifestyle Category 2 & $43(70)$ & $40(68)$ & $3(100)$ \\
$\quad$ Non-destroyed own house & $3(5)$ & $3(6)$ & 0 \\
Partially destroyed own house & $2(3)$ & $2(3)$ & 0 \\
Half destroyed own house & $2(3)$ & $2(3)$ & 0 \\
Massive destroyed own house & $2.0 \pm 1.8^{\#}$ & $1.9 \pm 1.8$ & $4.0 \pm 1.7$ \\
Completely destroyed own house & $1.2 \pm 0.9^{*}$ & $1.2 \pm 0.8$ & $2.7 \pm 1.5^{\$}$ \\
PTSD total score & $0.8 \pm 1.4^{*}$ & $0.7 \pm 14^{*}$ & $1.3 \pm 0.6$ \\
Category A+B & & \\
Category C & . & \\
\hline
\end{tabular}

${ }^{\#} \mathrm{P}<0.05$ vs. Graves' disease. ${ }^{*} \mathrm{P}<0.03$ vs. category $\mathrm{A}+\mathrm{B} .{ }^{\$} \mathrm{P}<0.01$ vs. Non-worsened group. The number in parenthese represents the percentage of each item in each group.

Table 9. Intensity grade of earthquake, lifestyles and psychological aspects in patients with central diabetes insipidus or adrenal insufficiency affected by the earthquake

\begin{tabular}{|c|c|c|c|c|c|}
\hline & \multirow{2}{*}{$\begin{array}{c}\text { Central diabetes } \\
\text { insipidus }\end{array}$} & \multicolumn{4}{|c|}{ Adrenal insufficiency } \\
\hline & & All & Panhypopituitarism & $\begin{array}{l}\text { ACTH isolated } \\
\text { deficiency }\end{array}$ & $\begin{array}{c}\text { Addison's } \\
\text { disease }\end{array}$ \\
\hline Number & 6 & 16 & 5 & 10 & 1 \\
\hline Age & $48 \pm 11 *$ & $68 \pm 16$ & $58 \pm 10$ & $73 \pm 11$ & 83 \\
\hline $\operatorname{Sex}(\mathrm{F} / \mathrm{M})$ & $5 / 1$ & $9 / 7$ & $4 / 1$ & $2 / 8$ & $1 / 0$ \\
\hline \multicolumn{6}{|l|}{ Therapy } \\
\hline DDAVP & 6 & & & & \\
\hline DDAVP, levothyroxine, hydrocortisone & & & 5 & & \\
\hline Hydrocortisone & & & & 10 & 1 \\
\hline Intensity of earth quake (JIS) & $6 \pm 0$ & $5.8 \pm 0.4$ & $5.8 \pm 0.4$ & $5.8 \pm 0.4$ & 6 \\
\hline \multicolumn{6}{|l|}{ Lifestyle Category 1} \\
\hline Own home & $6(100)$ & $15(94)$ & $4(80)$ & $10(100)$ & $1(100)$ \\
\hline Refuge house & 0 & $1(6)$ & $1(20)$ & 0 & 0 \\
\hline \multicolumn{6}{|l|}{ Lifestyle Category 2} \\
\hline Non-destroyed own house & $1(17)$ & $4(25)$ & $1(20)$ & $3(30)$ & 0 \\
\hline Partially destroyed own house & $5(83)$ & $8(50)$ & $2(40)$ & $6(60)$ & 0 \\
\hline Half destroyed own house & 0 & $2(13)$ & $1(20)$ & $1(10)$ & 0 \\
\hline Massive destroyed own house & 0 & $1(6)$ & 0 & 0 & $1(100)$ \\
\hline Completely destroyed own house & 0 & $1(6)$ & $1(20)$ & 0 & 0 \\
\hline PTSD total score & $2.3 \pm 1.4^{* *, \#}$ & $1.1 \pm 0.9^{\$}$ & $1.0 \pm 0.7$ & $1.0 \pm 0.7$ & 2 \\
\hline Category $\mathrm{A}+\mathrm{B}$ & $1.3 \pm 0.5$ & $1.0 \pm 0.7$ & $1.0 \pm 0.7$ & $0.9 \pm 0.7$ & 2 \\
\hline Category $\mathrm{C}$ & $1.0 \pm 0.9$ & $0.1 \pm 0.3^{*}$ & $0 *$ & $0.1 \pm 0.3^{*}$ & 0 \\
\hline
\end{tabular}

* $\mathrm{P}<0.01$ vs category A+B. ${ }^{* *} \mathrm{P}<0.01$ vs. adrenal insufficiency. ${ }^{*} \mathrm{P}<0.05$ vs. Graves' disease. ${ }^{\$} \mathrm{P}<0.05$ vs. Hashimoto's disease. The number in parenthesis represents the percentage of each item in each group. DDAVP represents desmopressin acetate.

ly higher than the mean of category $\mathrm{C}$; in patients with Graves' disease or CDI, there was no significant difference in the mean score of the combined categories A and B and the mean score of category C (Tables 7-9). In patients with Hashimoto's disease whose thyroid function had deteriorated, the mean score of the com- 
Table 10. Risk factor for the worsened thyroid functions in the patients with Graves' disease after the shock by multiple logistic regression analysis

\begin{tabular}{|c|c|c|c|}
\hline Predictor & Multivariate-adjusted odds ratio & $95 \% \mathrm{CI}$ & $P$ value \\
\hline Age (year) & 0.99 & 0.96 to 1.03 & 0.71 \\
\hline Sex & 1.19 & 0.31 to 4.60 & 0.80 \\
\hline FT3 (pg/ml) & 0.89 & 0.64 to 1.25 & 0.51 \\
\hline FT4 (ng/dl) & 1.36 & 0.51 to 3.65 & 0.54 \\
\hline $\mathrm{TSH}(\mu \mathrm{IU} / \mathrm{ml})$ & 1.03 & 0.99 to 1.08 & 0.15 \\
\hline TRAb (IU/l) & 1.02 & 1.00 to 1.04 & $0.003^{\#}$ \\
\hline TSAb (\%) & 1.00 & 1.00 to 1.00 & 0.63 \\
\hline \multicolumn{4}{|l|}{ Therapy } \\
\hline Thionamide & 1.06 & 0.97 to 1.16 & 0.18 \\
\hline${ }^{131}$ I therapy & 2.17 & 0.57 to 8.34 & 0.26 \\
\hline Intensity of earthquake (JIS) & 0.73 & 0.41 to 1.32 & 0.30 \\
\hline \multicolumn{4}{|l|}{ Lifestyle } \\
\hline Category 1 & 2.44 & 0.71 to 8.40 & 0.16 \\
\hline Category 2 & 1.56 & 0.86 to 2.84 & 0.14 \\
\hline \multicolumn{4}{|l|}{ PTSD score } \\
\hline Total score & 0.85 & 0.38 to 1.89 & 0.69 \\
\hline Category $\mathrm{C}$ & 0.65 & 0.17 to 2.52 & 0.53 \\
\hline
\end{tabular}

\# An alpha value of less than 0.05 was considered to be statistically significant. Values of FT3, FT4, TSH and TRAb, and ${ }^{131}$ I therapy before the shock were used as risk factors. The dose of thionamide received after the shock was also used as a risk factor. Lifestyle categories and PTSD score were obtained using questionnaire after the shock.

bined categories $\mathrm{A}$ and $\mathrm{B}$ was higher than the mean score of category C (Table 8).

The patient who interrupted her $L$-T4 treatment due to ${ }^{131} \mathrm{I}$ therapy for Graves' disease lived in a JIS grade 6 area, and her own house was partially destroyed. She sought refuge in another house. Nevertheless, this patient's PTSD score was zero. The other patient who interrupted her $L$-T4 treatment for hypothyroidism due to Hashimoto's disease lived in a JIS grade 7 area, and her own house was half destroyed. She sought refuge in another house, and her PTSD score was 4 (categories $\mathrm{A}$ and $\mathrm{B}, 2$; C, 2).

\section{3-4 Evaluation of risk factors for thyroid function deterioration after the earthquake}

Multiple logistic regression analysis demonstrated that, in patients with Graves' disease, the significant risk factor for thyroid function deterioration after the earthquake was serum TRAb titer (Table 10).

\section{Discussion}

The MNPE differed from the HAE [2] in several ways. First, the recorded maximum acceleration was much greater for the MNPE than for the HAE. Second, numerous strong aftershocks (JIS grade 5-6) continued for 2 months after the initial shock. Third, the MNPE occurred in a mountainous and rural area with villages, while the HAE struck an urban area. Fourth, 10 years had elapsed between the MNPE and the HAE, from which valuable lessons had been learned [2].

The differences in the consequences of the HAE and the MNPE can be explained by many factors. First, most people in the MNPE-affected area followed an agrarian lifestyle and tended to live cooperatively. Their houses are built to a higher standard due to the harsh climate conditions, and there is a greater distance between the houses than in the urban area affected by the HAE. Second, the MNPE occurred in the late autumn during fine weather that continued for 1 month, while the HAE occurred in the middle of winter during inclement weather. Third, more of the necessary equipment was available following the MNPE than following the HAE. Fourth, after the MNPE, the medical response of neighboring organizations was swifter and more substantial than that following the HAE (2). In addition, in contrast to the situation after the HAE, most medical facilities were able to continue functioning and were able to maintain communication with each other. Fortunately, we were able to provide medical supplies to those in need, and all patients except two 
were able to take their medication without interruption.

In the MNPE, our results show that none of the patients with thyroid diseases, CDI and AI developed a crisis after the shock. Moreover, the clinical status of most patients with Graves' or Hashimoto's diseases did not deteriorate. In addition, the PTSD score was much lower than that following the HAE [11, 12], although the PTSD score in the patients with CDI or Hashimoto's disease was higher than in the other patients.

Interestingly, although most patients with Graves' and Hashimoto's diseases were euthyroid after the initial shock, hyperthyroidism and hypothyroidism deteriorated in $19 \%$ of patients with Graves' disease and in $5 \%$ of patients with Hashimoto's disease.

In the patients with Graves' disease, the incidence of thyroid function deterioration increased with greater severity of hyperthyroidism before the earthquake. Based on the findings of multiple logistic regression analysis and a comparison of the patients whose thyroid function had deteriorated with that of patients whose thyroid function remained stable, serum TRAb titer was identified as the risk factor for thyroid function deterioration. It is likely that some patients with high serum TRAb titers may be more sensitive than patients with low serum TRAb titers to earthquakeinduced stress, which drives the immune system towards a Th-2 response [6].

Subclinical hypothyroidism was found after the earthquake in a few patients with Hashimoto's disease. The reason for this finding is not clear. However, as there does not appear to be a direct relationship between stress and Hashimoto's disease [6], some patients, such as the two patients who had hypothyroidism, may have temporarily stopped taking their $L$-T4, even though they did not report this on the questionnaire. The total PTSD and the environmental effects scores were higher in Hashimoto's disease patients who developed subclinical hypothyroidism than in those whose thyroid function remained stable, suggesting that the quality of life of patients whose thyroid function had deteriorated may have been adversely affected, making it impossible for them to take their $L$-T4 regularly after the earthquake.

The mean PTSD scores in patients with CDI and Hashimoto's disease were different from those of the other patients. CDI patients were younger than the AI patients, while Hashimoto's disease patients were older than the Graves' disease patients. Interestingly, the degree of worsening of mental status caused by the earthquake in Hashimoto's disease or AI patients was lower than the environmental effects caused by the earthquake. However, there was no difference between the two in patients with Graves' disease or CDI. The different pathophysiological conditions in Graves' and Hashimoto's diseases may explain the different status. Furthermore, following the HAE, patients' conditions were found to be related to the degree of activity of the HPA axis [12]. In patients with AI, the HPA axis could have been inactivated by stress due to a disturbance of the HPA axis, while the HPA axis in CDI patients may have been activated by stress, even though their neurohypophyseal function was disturbed [13]. Thus, a difference in HPA axis activity could result in a higher PTSD score, so that CDI patients would have a worse mental status than AI patients.

Our findings suggest that, in patients with Graves' disease, it is necessary to maintain their euthyroid state with low serum TRAb titers to prevent thyroid function deterioration after an earthquake, because patients with a high serum TRAb titer may have immune systems that are more sensitive towards a $\mathrm{Th}-2$ response due to the earthquake. In fact, all patients should continue to take their medication after an earthquake, since it is likely that their lives are interrupted by environmental effect owing to earthquake-shock, especially patients with CDI or Hashimoto's disease. Thus, due to the risk of medical facility closure during a disaster, all patients should always have a note or copy of their medical records, including medical history and medications that are being used, to avoid relying on the patients remembering the names and doses of their medications.

In conclusion, all patients should continue to take their medication following an earthquake. To help achieve this objective, neighboring organizations must respond quickly with sufficient medical support for patients with endocrine disorders following an earthquake, and the patients affected by a strong earthquake should quickly obtain medical care. In order to obtain maximum benefit from the available medical care, patients should always have a note or copy of their medical records, including their medical history and their medications. In addition, appropriate information should be provided to all affected people, in particular those with AI, using all means possible, including the mass media, to decrease any adverse consequences.

This abstract was presented at the 79th Annual Meeting of the Japan Endocrine Society, Tokyo, and May 19, 2006. 


\section{References}

1. The Earthquake Engineering Research Institute (2005) Special Earthquake Report - January 2.

2. Baba S, Taniguchi H, Nambu S, Tsuboi S, Ishihara K, Osato S (1996) The Great Hanshin earthquake. Lancet 347: 307-309.

3. Matsuoka T, Yoshida T, Oda J, Tanaka H, Kuwagata Y, Sigimoto H, Sigimoto T (2000) The impact of a catastrophic earthquake on morbidity rates for various illnesses. Public Health 114: 249-253.

4. Besdovsky HO, del Rey A (1996) Immune-neuroendocrine interactions: facts and hypothesis. Endocr Rev 17: 64-102.

5. Eskandari F, Sternberg EM (2002) Neural-immune interactions in health and disease. Ann NY Acad Sci 966: 20-27.

6. Prummel FM, Striender T, Wiersinga MW (2004) The environment and autoimmune thyroid diseases. Eur $J$ Endocrinol 150: 605-618.

7. Research group for Hypothalamo-pituitary Disorders (2001) Guidelines for the Diagnosis and Treatment of Hypothalamo-pituitary Disorders. Ministry of Health, Labour and Welfare (in Japanese).

8. The Japan Thyroid Association. Guidelines for the
Diagnosis and Treatment of Thyroid Disorders. http:// thyroid.umin.ac.jp

9. Ladenson PW, Singer PA, Ain KB, Bagchi N, Bigos ST, Levy EG, Smith SA, Daniels GH, Cohen HD (2000) American Thyroid Association guidelines for detection of thyroid dysfunction. Arch Int Med 160: 1573-1575.

10. American Psychiatric Association. Diagnostic and statistical manual of mental disorders. Washington, DC: APA, 1995; $4^{\text {th }}$ ed.

11. Fukuda S, Morimoto K, Mure K, Maruyama S (1999) Posttraumatic stress and change in lifestyle among the Hanshin-Awaji earthquake victims. Prev Med 29: 147151.

12. Fukuda S, Morimoto K, Mure K, Maruyama S (2000) Effect of the Hanshin-Awaji earthquake on posttraumatic stress, lifestyle changes, and cortisol levels of victims. Arch Environ Health 55: 121-125.

13. Zelena D, Foldes A, Mergl Z, Barna I, Kovacs KJ, Makara GB (2004) Effects of repeated restraint stress on hypothalamo-pituitary-adrenocortical function in vasopressin deficient Brattleboro rats. Brain Res Bull 63: 521-530. 